\title{
Scanning tunneling microscopy of InAs/GaSb superlattices: Subbands, interface roughness, and interface asymmetry
}

\author{
R. M. Feenstra \\ IBM Research Dirision. T. J. Watson Research Center, Yorktown Heights, New York 10598
}

D. A. Collins, D. Z.-Y. Ting, M. W. Wang, and T. C. McGill

T. J. Watson Sr. Laboratory of Applied Physics, California Institute of Technology. Pasadena, California 91125

(Received 25 January 1994; accepted 12 March 1994)

\begin{abstract}
Scanning tunneling microscopy and spectroscopy is used to characterize InAs/GaSb superlattices, grown by molecular-beam epitaxy. Roughness at the interfaces between InAs and GaSb layers is directly observed in the images, and a quantitative spectrum of this roughness is obtained. Electron subbands in the InAs layers are resolved in spectroscopy. Asymmetry between the interfaces of $\operatorname{InAs}$ grown on GaSb compared with GaSb grown on InAs is seen in voltage-dependent imaging. Detailed spectroscopic study of the interfaces reveals some subtle differences between the two in terms of their valence-band onsets and conduction-band state density. These differences are interpreted in a model in which the GaSb on InAs interface has an abrupt InSb-like structure, but at the InAs on GaSb interface some Sb grading occurs into the InAs overlayer.
\end{abstract}

\section{INTRODUCTION}

The InAs/GaSb superlattice system has properties which are both scientifically interesting and useful for applications. $^{1-5}$ This system, as well as the related InAs/ AlSb system. is a type II superlattice, with electrons confined in the InAs layers and holes in the GaSb layers. As shown in Fig. 1, the conduction-band minimum $\left(E_{C}^{\Gamma}\right)$ of bulk InAs lies below the valence-band maximum $\left(E_{V}\right)$ of bulk GaSb, which would result in transfer of electrons from $\mathrm{GaSb}$ to In As if the bulk materials were brought into contact. In narrow superlattice layers, however, quantum confinement shifts the energy levels so that the lowest electron subband in InAs is above the highest hole subband in GaSb. Transitions between these levels, separated by $\sim 0.1 \mathrm{eV}$, are used for infrared photodetectors (generally made with $\mathrm{Ga}_{1-x} \mathrm{In}_{x} \mathrm{Sb}$ alloys rather than pure GaSb). ${ }^{\prime}$ An important issue in these devices is the precise chemistry of the interfaces: Since the constituent materials have different anions and cations, the interface composition may be Ga bonded to As(GaAs-like). In bonded to $\mathrm{Sb}($ InSb-like), or some intermediate phase. The interface composition is predicted to affect device behavior. ${ }^{2}$ In addition, interface roughness is also expected to be an important parameter in device performance (e.g., in determining mobility), and thus it is desirable to obtain a quantitative determination of the roughness.

In this work, we present results from cross-sectional scanning tunneling microscopy (STM) studies of InAs/GaSb superlattices. Detailed spectroscopic studies are used to reveal the electron subband structure in the InAs, and to probe the chemistry at the semiconductor interfaces. We find a distinct asymmetry between the interfaces for InAs grown on GaSb compared with GaSb grown on InAs, attributed in the former case to $\mathrm{Sb}$ incorporation in the InAs overlayer. In addition, interface roughness is directly observed in the STM images, and quantitative spectrum of the roughness is obtained.

This article is organized as follows. Section II describes the experimental details, including growth conditions for the superlattices. STM images are presented in Sec. III, and analysis of the interface roughness is described in detail. Spectroscopic results are given in Sec. IV, including a comparison of superlattice spectra with those obtained from bulk material, and a discussion of the interface asymmetry as seen in voltage-dependent imaging and spatially resolved spectroscopy.

\section{EXPERIMENT}

The InAs/GaSb superlattices were grown on GaSb substrates using a Perkin-Elmer 430 molecular-beam epitaxy (MBE) system, at growth temperatures of $\sim 380^{\circ} \mathrm{C}$. The growth surface was exposed to an $\mathrm{Sb}_{2}$ flux for $5 \mathrm{~s}$ at the termination of each InAs and GaSb layer. The superlattices are $\mathrm{Si}$ doped at concentrations of $3 \times 10^{17} \mathrm{~cm}^{-3} p$ type in GaSb and $3 \times 10^{17} \mathrm{~cm}^{-3} n$ type in InAs. The intended superlattice period. based on growth times calibrated to prior reflection high-energy electron diffraction (RHEED) studies, was 15 bilayers of InAs and 8 bilayers of GaSb, 3.0 $\AA$ per bilayer, ${ }^{7}$ for a total thickness of $69 \AA$. Subsequent $x$-ray rocking curves yielded a period of $67 \pm 1 \AA$. From a detailed analysis of the STM images, we find that the superlattice period corresponds to $22 \pm 0.5$ bilayers $=66 \pm 1.5 \AA$, which is consistent with the $x$-ray analysis, but one bilayer less than the intended period. For the purposes of theoretical simulation, it is convenient to use an integer number of bilayers for each period, so we must choose between $(14,8)$ or $(15,7)$ bilayers of (InAs, GaSb). The spatially resolved spectroscopic results below are more consistent with the former, so we take $(14,8)$ bilayers as the nominal superlattice period. Two wafers have been studied, using multiple samples from each wafer, and similar results were obtained on all cleaves. Samples cut from the wafers were cleaved in situ, at pressure of $4 \times 10^{-11}$ Torr. Single crystal $\langle 111\rangle$ oriented tungsten probe tips were prepared by electrochemical etching and $i n$ situ electron beam heating, and were characterized in situ by field-emission microscopy. STM images were acquired with 


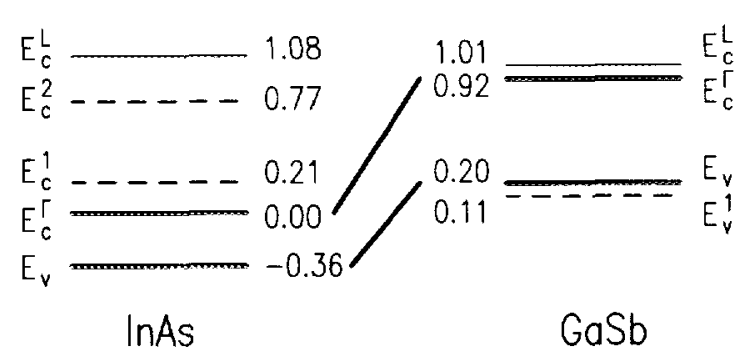

FiG. 1. Room-temperature energy levels for lnAs and GaSb, in eV, from Ref. 6. Bulk valence-band maxima are denoted by $E_{V}$. Direct, $\Gamma$-valley conduction-band minima are denoted by $E_{C}^{\prime}$, and $L$-valley minima are denoted $E_{C}^{L}$. Quantum subbands for a superlattice containing 42 - $\mathrm{A}$-thick $\ln$ As layers and $24-\AA$-thick GaSb layers are shown: the first two electron subbands are denoted $E_{C}^{1}$ and $E_{C}^{2}$, and the first hole subband is denoted $E_{V}^{1}$. The zero of energy is taken to be $E_{C}^{\top}$ of $\operatorname{In} A$ s.

constant current of $0.1 \mathrm{nA}$ and at various voltages specified below. Spectra from bulk materials were obtained using $2 \times 10^{17} \mathrm{~cm}^{-3} p$-type GaSb and $9 \times 10^{17} \mathrm{~cm}^{-3} n$-type InAs crystals.

Spectroscopic measurements were performed using a method previously developed for obtained a large dynamic range in the tunnel current and conductance. ${ }^{8,9}$ Briefly, the tip-sample separation is varied as the voltage is scanned according to $\Delta s=+a|V|$, with $a \simeq 1 \AA / \mathrm{V}$. Subsequent normalization is accomplished by computing the ratio of differential to total conductance $(d I / d V) / \overline{(I / V)}$, where the total conductance $\overline{I / V}$ is broadened over an energy range of $1.5 \mathrm{~V}$ to form a suitable normalization quantity. Dynamic conductance is measured using a lock-in amplifier, with typically $50-\mathrm{mV}$ modulation on the bias voltage.

The precision of energetic positions determined by tunneling spectroscopy can, in some cases, be limited by tipinduced band bending in the semiconductor. ${ }^{10.11}$ For states which are strongly localized at the surface, the error is given by the amount of voltage which is dropped in the depletion layer of the semiconductor, which can be several tenths of an $\mathrm{eV}$ for doping levels near $10^{18} \mathrm{~cm}^{-3}$ and applied biases $\leqslant 1$ V. However, for the case of bulk derived spectroscopic features such as band onsets, the effects of tip-induced band bending are significantly reduced due to tunneling of the carrier through the depletion region of the semiconductor. ${ }^{10}$ Corrections to the observed energies are then $\leqslant 0.1 \mathrm{eV}$ for doping levels near $10^{18} \mathrm{~cm}^{-3}$. For superlattice spectroscopy discussed below, band bending effects are reduced even further because the superlattice band gap is so small. Thus, tip-induced band bending does not significantly affect our spectroscopic results.

\section{STRUCTURAL ANALYSIS}

In Fig. 2 we show a large-scale STM image of the InAs/ $\mathrm{GaSb}$ superlattice. Here, as in all our images, the superlattice growth direction is to the left. We see in Fig. 2 the GaSb buffer layer on the right-hand side, followed by a series of In $\mathrm{As} / \mathrm{GaSb}$ superlattice periods, with the GaSb layers appearing bright and the InAs layers dark (the origin of this contrast is explained in Sec. IV). The apparent curvature at the top of

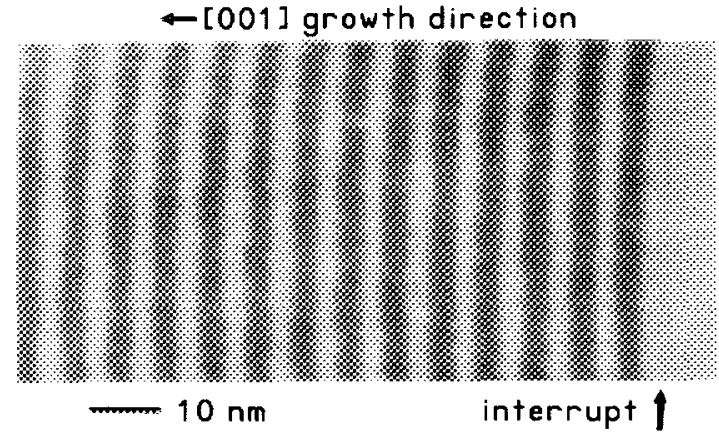

Fig. 2. Large-scale STM image of an InAs/GaSb superlattice, consisting of 42- $\AA$-thick InAs and $24-\AA$-thick GaSb layers. The superlattice was grown on a $\mathrm{GaSb}$ butfer layer, seen on the right side of the image. A growth interrupt near the top of this buffer layer is marked by an arrow. The image was acquired at a sample voltage of $-1.3 \mathrm{~V}$. The [001] growth direction is indicated. Grey-scale range is $2.8 \AA$. The curvature seen near the top of the image is due to drift of the STM.

the image is due to some drift in the STM during image acquisition. Note the small dark line in the image, just to the right of the first InAs layer: this line signifies some slight electronic disturbance in the material, arising from a growth interrupt which was performed (to check the RHEED pattern) prior to growing the superlattice.

Figure 3 shows an expanded view of the superlattice. Faint fringes, with spacing of $6.0 \AA$, are visible in the image, seen most clearly in the InAs layers. These fringes arise from the lattice planes; the bilayer spacing is $3.0 \AA$, and every second bilayer is resolved at the surface. Apparent in both Figs. 2 and 3 is the presence of roughness at the interface between the InAs and $\mathrm{GaSb}$ layers. This roughness can be better defined as follows. We expand the grey scale of the image in Fig. 3, and then take a derivative in the horizontal direction, producing the image shown in Fig. 4. The interface positions in that image are distributed over a few pixels, and

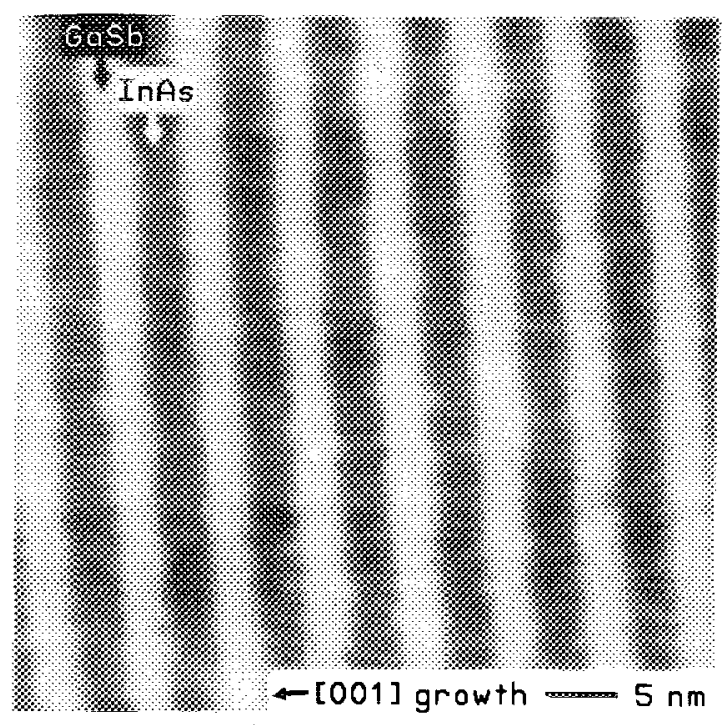

FIG. 3. STM image of the InAs/GaSb superlattice, indicating the InAs layers (dark) and GaSb layers (bright). The image was acquired at a sample voltage of $-1.3 \mathrm{~V}$. Grey-scale range is $2.1 \AA$ 


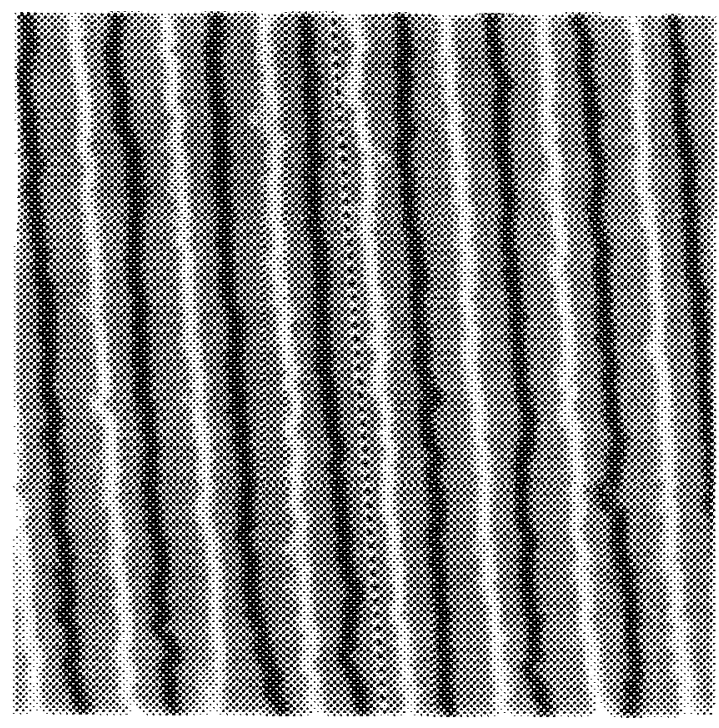

Fici. 4. Analyzed data, consisting of a derivative of the image of Fig. 3 Interface profiles for InAs grown on GaSb appear as bright lines. and for GaSt on InAs as dark lines. The base line used for protile measurement is shown by the dashed line.

their precise locations are obtained by computing the mean value of each of these distributions. We then obtain the interface profiles. $A(x)$, by measuring the perpendicular separation between the interface and some base line, as a function of distance $x$ along the base line. Our choice of base line. corresponding to the average slope of the interfaces, is shown by the dashed line in Fig. 4 .

As seen in Fig. 4, the interface roughness has the form of 1-2 bilayer high steps. separated by distances ranging from 10 to $100 \AA$ along an interface. To quantify the roughness. we Fourier transform the profiles according to

$$
A_{q}=\frac{1}{, L} \int_{-L / 2}^{L / 2} A(x) e^{-i q x} d x \text {, }
$$

where $q$ is the wave vector and $L$ is the length of interface being sampled. The resulting Fourier spectra are shown in Fig. 5, averaging over the seven full length (500 $\AA$ long) interfaces of each type from Fig. 4. We see in Fig. 5 that the roughness for the two types of interfaces is similar, although the $\mathrm{GaSb}$ on InAs interface displays slightly higher roughness at low wave vectors. To quantify the roughness. we fit the spectra to a suitable functional form, which we choose to be a Lorentzian.

$$
\left|A_{q}\right|^{2}=\frac{2 \Lambda \Delta^{2}}{1+q^{2} \Lambda^{2}}
$$

where $\Delta$ is the roughness amplitude and $\Lambda$ is the correlation length. The Fourier transform of this equation gives an exponential real-space correlation function,

$$
\left\langle A(x) A\left(x^{\prime}\right)\right\rangle=\Delta^{2} e^{-1,-x^{\prime} \mid x .} \text {. }
$$

This form for the correlation function is that expected for a random distribution of steps with average spacing $\Lambda$. and

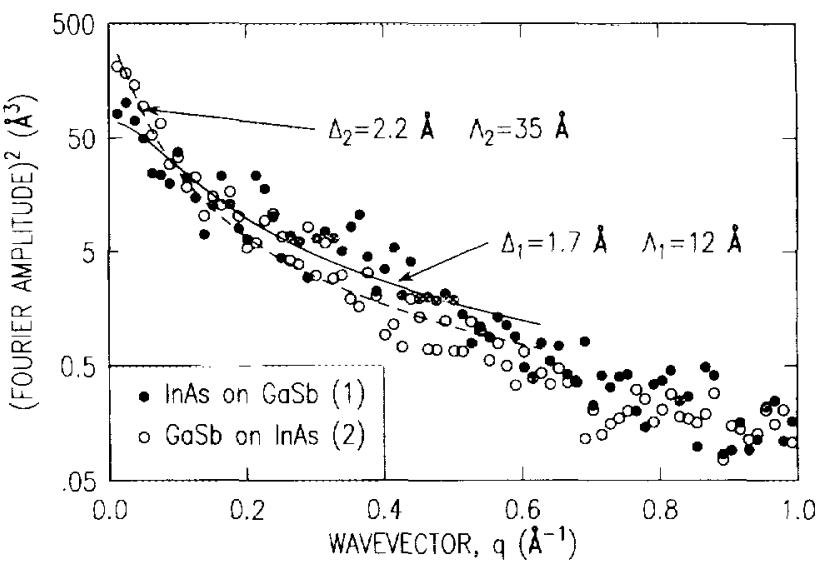

FIG. 5. Interface roughness spectrum. computed on interfaces of length $L=500 \AA$, using seven interfaces of each type (from Fig. 4 l and averaging the results. The data from each interface type are fit to a Lorentzian form, yielding results shown for the roughness amplitude $(\Delta)$ and correlation length (A).

thus it is well suited to the present study of interface roughness. The resulting Lorentzian frequency distribution for the roughness spectrum has substantial components extending out to high frequencies, in agreement with the our observed data. (High-frequency components were also observed in a roughness study of $\mathrm{Si} / \mathrm{SiO}_{2}$ interfaces.) ${ }^{12}$ Alternatively, the conventional assumption of a Gaussian distribution for the roughness spectrum ${ }^{13}$ provides a poor fit to the data.

Fitting Eq. (2) to the spectra in Fig. 5 yields parameters of $\Delta_{1}=1.7 \AA$ and $\Lambda_{1}=12 \AA$ for the InAs on GaSb interfaces, and $\Delta_{2}=2.2 \AA$ and $\Lambda_{2}=35 \AA$ for the GaSb on InAs interfaces. The Lorentzian form provides a good fit over the wave vector range $0-0.6 \AA^{-1}$, although at higher wave vectors the data decrease somewhat faster than the fit. The above values for the roughness amplitudes correspond approximately to the rms roughness of the interfaces, given by

$$
\left\langle|A(x)-\bar{A}|^{2}\right\rangle=\frac{1}{L} \sum_{q \neq 0}\left|A_{q}\right|^{2}
$$

where $\bar{A}$ is the average value of each profile. We find rms values of $1.9 \AA$ for the InAs on GaSb interfaces, and $2.3 \AA$ for the GaSb on InAs interfaces. This difference between the rms roughness of the two types of interfaces is relatively small, and it arises from the low-frequency components of the roughness as noted above. A more significant measure of the difference between the types of interfaces is obtained by examining the correlation lengths. We find that correlation lengths for InAs on GaSb interfaces are consistently 2-3 times less than for $\mathrm{GaSb}$ on InAs, thus implying that the former is considerably rougher. This extra roughness has the form of closely spaced steps, which can be seen in a careful examination of Fig. 4. We thus conclude that the InAs on $\mathrm{GaSb}$ interfaces are rougher than $\mathrm{GaSb}$ on InAs, thus implying some differences in the growth characteristics for these interfaces. We note that the values of the roughness ampli- 
(a)

(b)

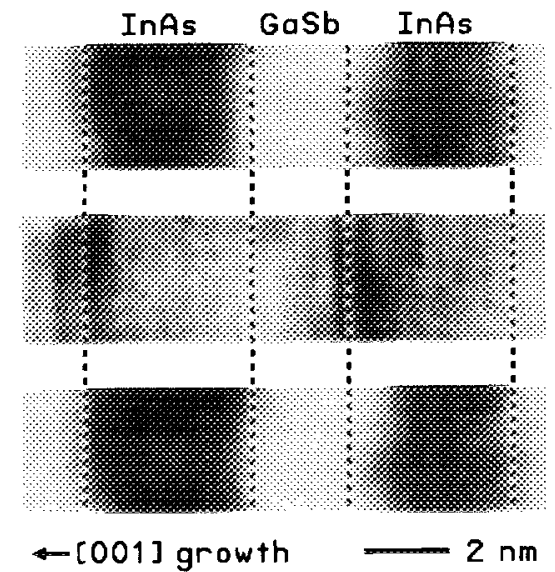

FIG. 6. Constant-current STM images, acquired at sample voltage of (a) -1.3 , (b) +1.3 , and (c) $-1.3 \mathrm{~V}$. Grey-scale ranges are (a) 1.4 , (b) 0.8 , and (c) $1.4 \AA$. Dashed lines indicate the position of the interfaces between $\ln A s$ and $\mathrm{GaSb}$

tudes and correlation lengths obtained in our analysis are in the range to be important in determining the mobility of the superlattices. ${ }^{14}$

\section{SPECTROSCOPIC ANALYSIS}

In Sec. III we discussed filled state STM images, in which the GaSb layers appeared brighter (greater tip-sample separation for constant current, or equivalently, greater tunnel current for fixed separation) than the InAs layers. The origin of this contrast arises simply from the band offset in the system: Since the valence-band maximum in $\mathrm{GaSb}$ is 0.56 $\mathrm{eV}$ higher than that of InAs, and the valence-band shapes are similar in the two materials, then at a given negative sample bias the tunnel current from $\mathrm{GaSb}$ will be substantially greater than from InAs. The topographic transition between the layers was used to define the interface position, as in Fig. 4. The situation for the empty states turns out to be more complicated. As pictured in Fig. 1, states from the $\Gamma$-valley conduction band of InAs are much lower in energy than those of $\mathrm{GaSb}$, but states from the $L$ valleys in the materials are at nearly the same energy. The positive sample biases used for imaging are generally large enough to include the $L$ valleys, in which case the tunnel currents from the InAs and $\mathrm{GaSb}$ layers are comparable. In this case however, we find distinct differences in the image contrast at the interfaces between InAs and GaSb, as described below.

Voltage dependence of the STM images is shown in Fig. 6, where images (a)-(c) were acquired consecutively on the same surface region. The filled state images, Figs. 6(a) and 6(c) are similar to Fig. 3. The empty state image, Fig. 6(b), appears much different, with an enhanced signal (greater state density) at the InAs on GaSb interfaces compared with $\mathrm{GaSb}$ on InAs. The large asymmetry seen between the two types of interfaces in Fig. 6 demonstrates that there is some significant structural difference between them. The STM images indicate an ideal, epitaxial structure at both types of interfaces, so the observed asymmetry probably involves a

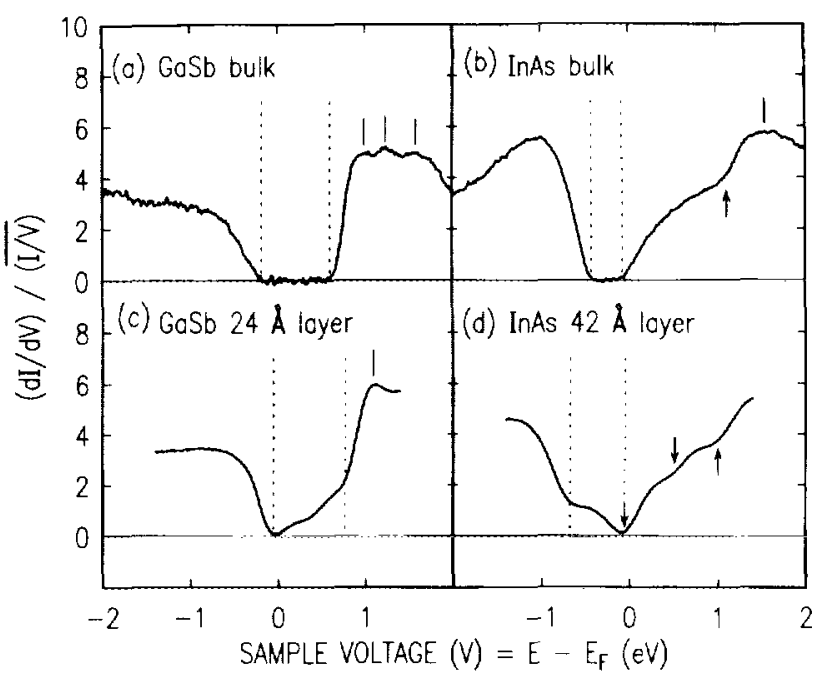

FIG. 7. Typical tunneling spectra, acquired from (a) bulk GaSb, (b) bulk InAs, (c) GaSb superlattice layer. and (d) InAs superlattice layer. Apparent band gaps are indicated by dashed lines, surface states by tic marks, and $L$-valley conduction band onsets by upward pointing arrows. Downward pointing arrows indicate subband onset energies in panel (d).

difference in the chemistry of the interface bonding. To further explore this asymmetry, we perform detailed spectroscopic measurements.

As an introduction to the spectroscopy, we first examine typical spectra acquired near the center of the superlattice layers compared with those obtained from bulk InAs and $\mathrm{GaSb}$, as shown in Fig. 7. The bulk spectra are part of a larger study, which also includes detailed spectroscopy of InSb, InP, and GaAs. Spectral features are indicated in Fig. 7 , where the energetic positions are determined using peak locations for surface states, and assuming linear onsets for the bulk bands, with a precision of $\pm 0.03 \mathrm{eV}$. Energies for band gaps and subbands quoted below involve differences between observed onsets, and thus have an uncertainty of $\pm \sqrt{2(0.03)}$ $\simeq \pm 0.04 \mathrm{eV}$. For bulk GaSb, Fig. 7(a), we find a band gap of $0.78 \mathrm{eV}$ (compared with known value of $0.72 \mathrm{eV}$ ) and surface state locations in good agreement with inverse photoemission results. ${ }^{15}$ For bulk InAs, we find a band gap of 0.35 $\mathrm{eV}$ and $L$-valley onset located $1.18 \mathrm{eV}$ above the $\Gamma$-valley minimum (compared with known values of 0.36 and $1.08 \mathrm{eV}$, respectively).

Turning now to the spectra acquired from the superlattice layers, Figs. $7(\mathrm{c})$ and $7(\mathrm{~d})$, we see features which are different than for the bulk materials. First, significant conductance is observed within the apparent band gap regions. Based on the intensity and spatial dependence of this feature, we interpret it as arising from electron (hole) states tailing out from neighboring InAs (GaSb) layers. Second, we find that the apparent band gaps are slightly larger for the superlattice compared with the bulk, with observed gaps of 0.82 and 0.62 $\mathrm{eV}$ for the GaSb and InAs layers, respectively. We attribute the significantly larger gap for InAs to confinement effects in the quantum well (this effect is smaller for GaSb due to the much larger heavy hole mass); subtracting the known InAs gap yields an energy for the first electron subband of $0.26 \mathrm{eV}$. The third feature seen in the superlatice spectra is an addi- 

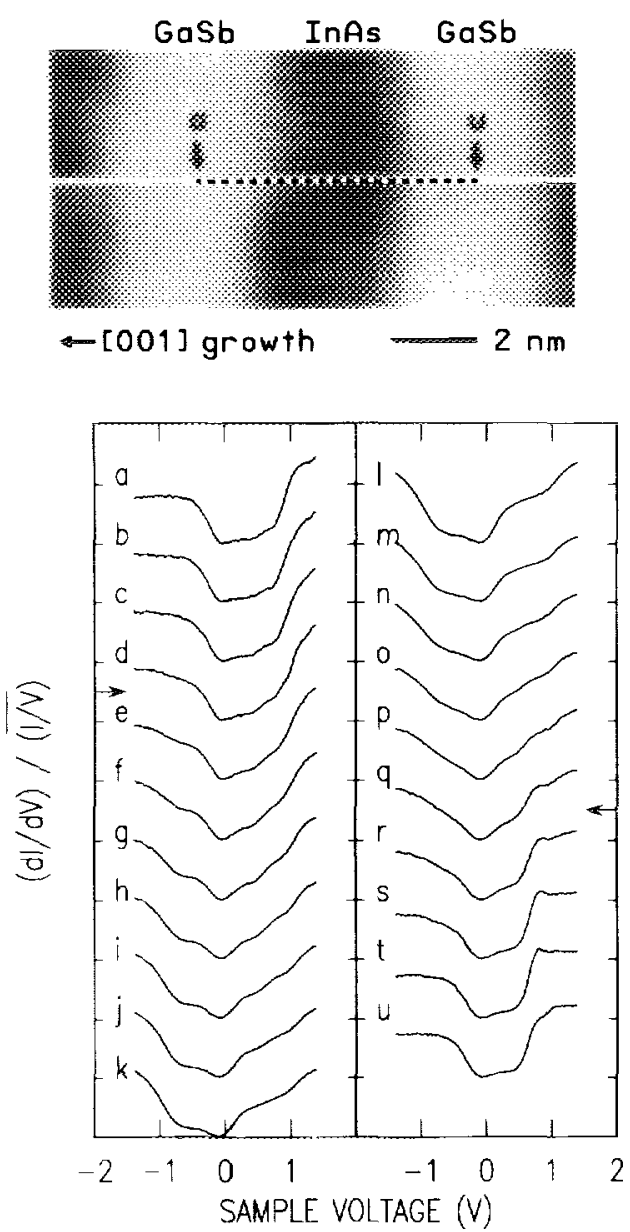

FIG. 8. Spatially resolved spectroscopy of InAs/GaSb superlattice. Spectra were acquired every $1.06 \AA$ along the dashed line shown in the middle of the STM image, with 63 of these spectra extending over one superlattice period. Every third spectrum, labeled a-u consecutively. is shown. The interface of GaSb grown on InAs occurs between spectra $d$ and $e$, and the interface of InAs yrown on GaSb occurs between $q$ and $r$.

tional onset at $0.51 \mathrm{~V}$, which we attribute to the second electron subband. Relative to the observed InAs valence-band edge, it is located at $1.18 \mathrm{eV}$; and subtracting the InAs bulk gap yields an energy of $0.82 \mathrm{eV}$. These subband energies $(0.26$ and $0.82 \mathrm{eV})$ are in reasonable agreement with theoretical estimates based on an eight-band tight binding model ${ }^{16}$ of 0.21 and $0.77 \mathrm{eV}$, thus confirming the above identification.

Spatially resolved spectroscopy results are displayed in Fig. 8. The STM image shown there was acquired in two halves; the bottom part first, followed by a set of spectra measured each $1.06 \AA$ along the dashed line, followed by the top half of the image. Correspondence between the upper and lower halves of the image demonstrate the absence of STM drift during acquisition of the spectra. The spacing between adjacent spectra is smaller than the STM resolution, so in Fig. 8 we display every third spectrum, labeled a-u consecutively. The spectra provide a detailed view of the spatially varying electronic structure in the superlattice. We see that the spectra vary from GaSb-like $(a-c)$, through the GaSb-on-InAs interface $(d-f)$, to InAs-like $(g-o)$, through
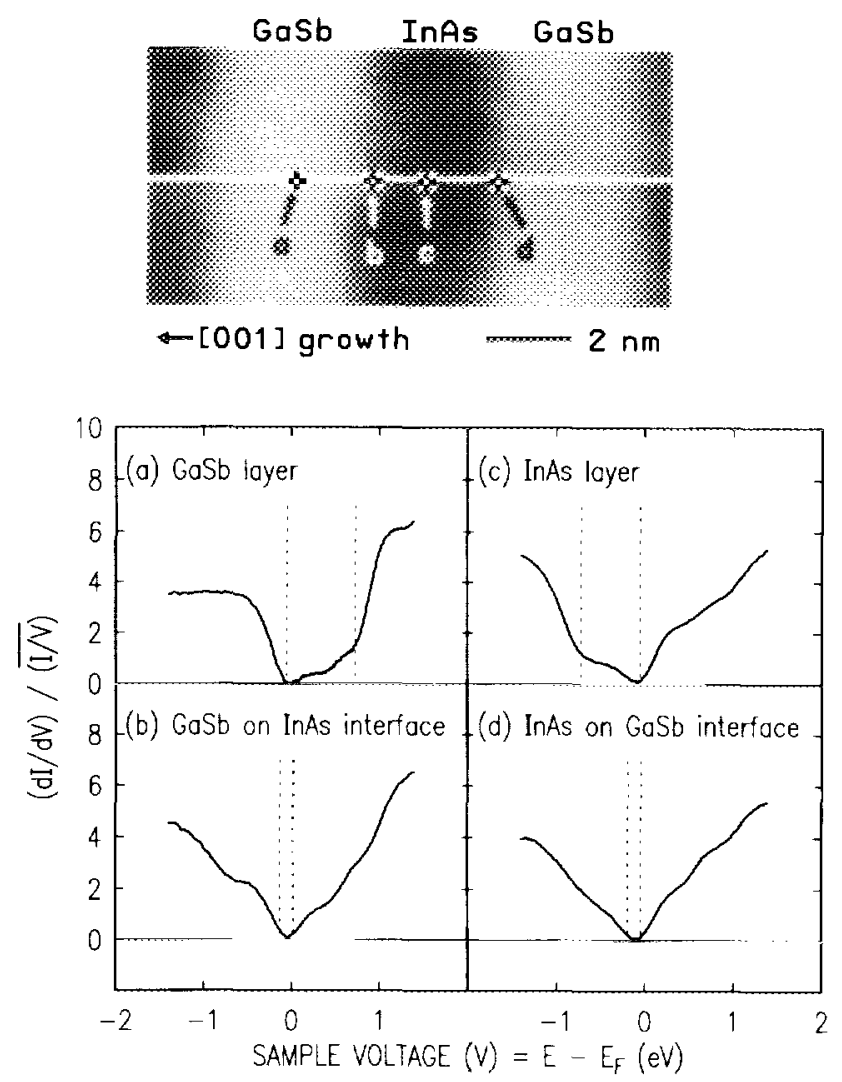

FIG. 9. Spatially resolved spectra selected at particular points in the InAs/ GaSb superlattice: (a) center of GaSb layer, (b) GaSb on InAs interface, (c) center of InAs layer, and (d) InAs on GaSb interface. Superlattice band gap is indicated by dashed lines in spectra (b) and (d).

the InAs-on-GaSb interface (p-r), and back to GaSb-like ( $\mathrm{s}-$ u). Spectra acquired near the center of the superlattice layers appear similar to those in Figs. 7(c) and 7(d). We note that the onset of the second electron subband, near $0.51 \mathrm{~V}$, is better defined at the edges of the $\ln A s$ layer $(e-h$ and $o-p$ ) compared with the center $(j-n)$, which we attribute to the presence of a central node for this subband (some asymmetry in the spectra between the two sides of the InAs layer is also evident, as discussed below). At the interfaces, we observe an enhanced intensity of the conductance near $\pm 0.5 \mathrm{~V}$ discussed above, consistent with its interpretation as arising from wave-function tails from adjoining layers.

In Fig. 9 we focus in on specific spectra, comparing those acquired near the center of the superlattice layers with those from the interfaces. The apparent band gap for the interface spectra. Figs. 9(b) and 9(d) is now marked as the minimum gap between empty and filled states, observed to be $0.1-0.2$ $\mathrm{eV}$ (compared with theoretical value of $0.15 \mathrm{eV}$ ). Closely inspecting the interface spectra, we find two differences between them: (1) for InAs on GaSb, the spectrum is smeared out, especially on the valence-band side, and (2) the normalized conductance is higher at low positive voltage for InAs on GaSb. This latter feature leads to the enhanced empty state density seen for that interface in Fig. 6. Although these features are relatively small, we emphasize that they are reproducible; both features have been seen in about 20 data

J. Vac. Sci. Technol. B, Vol. 12, No. 4, Jul/Aug 1994 
sets acquired from two samples, each sample studied with a different probe tip.

The above observation of spectral smearing at the InAs on $\mathrm{GaSb}$ interface is found to persist over a distance of $\sim 6 \AA$ (two bilayers). We thus propose that some mixing or grading occurs at this interface over this distance. This finding is consistent with both the enhanced roughness of these interfaces found in Sec. III, and with the interface structure deduced from growth studies; the GaSb growth is terminated by soaking the surface for $5 \mathrm{~s}$ with $\mathrm{Sb}_{2}$, thus forming a $1 \times 5$ reconstructed $\mathrm{Sb}$ layer. Photoemission [x-ray photoelectron spectroscopy (XPS)] studies reveal that some of this interfacial $\mathrm{Sb}$ rides up on the surface of the subsequently deposited InAs layer, along with a small amount of $\mathrm{Sb}$ incorporation into the InAs. ${ }^{3}$ Exchange of $\mathrm{Sb}$ with $\mathrm{As}$ at the InAs on $\mathrm{GaSb}$ interface may also occur, thus forming some GaAs-like interface bonds. We conclude that the InAs overlayer contains $\mathrm{Sb}$, in 1-2 bilayers from the InAs on GaSb interface. This conclusion is supported by the observation that, after starting the InAs growth, it takes 2-4 bilayers for the RHEED pattern to stabilize. ${ }^{3}$ For the case of GaSb on InAs, our STM results and the growth studies indicate a more abrupt interface, with InSb-like bonds. Additional measurements are underway, on interfaces prepared under a range of growth conditions, in an effort to further identify the interface chemistry.

\section{SUMMARY}

In summary, we have used a new method to deduce interface roughness in InAs/GaSb superlattices, and have studied intermixing at the interfaces using tunneling spectroscopy. The method developed here for roughness determination should have general applicability to a wide class of III-V heterointerfaces, which can serve to quantify many of the prior discussions concerning roughness in these systems. ${ }^{17-19}$ We find that our InAs on $\mathrm{GaSb}$ interfaces are rougher than $\mathrm{GaSb}$ on InAs, thus implying some difference between the growth characteristics for the interfaces. An asymmetry between the interfaces is also seen in voltage dependent imaging and spectroscopy, where we find that tunneling spectra at the InAs on GaSb interfaces display some spectral smearing. This spectral smearing is consistent with the enhanced roughness of the interfaces. Based on XPS and RHEED growth studies, we propose that these features arise from some $\mathrm{Sb}$ diffusion in the InAs overlayer, which occurs due to the surfactantlike nature of $S b$ which drives the $S b$ to the top of the InAs layer. Alternatively, the GaSb on InAs interfaces are found to be relatively abrupt, based on both the STM results and the growth studies.

\section{ACKNOWLEDGMENTS}

The authors gratefully acknowledge H. Munekata for providing us with some InAs wafers. This work was supported in part by the Advanced Research Projects Agency under Contract No. N00014-93-1-0881, and by the Air Force Office of Scientific Research under Contract No. F49620-93-10258 .

D. L. Smith and C. Mailhiot. J. Appl. Phys. 62. 2545 (1987); C. Mailhio and D. L. Smith. J. Vac. Sci. Technol. A 7. 445 (1989).

${ }^{2}$ R. H. Miles, J. N. Schulman, D. H. Chow, and T. C. McGill, Semicond Sci. Technol. 8, S102 (1993).

${ }^{3}$ D. A. Collins, T. C. Fu. T. C. McGill, and D. H. Chow. J. Vac. Sci Technol. B 10, 1779 (1992); M. W. Wang. D. A. Collins, T. C. McGill. and R. W. Grant, ibid. 11. 1418 (1993)

${ }^{4}$ B. R. Bennett, B. V. Shanabrook. R. J. Wagner. J. L. Davis, and J. R. Waterman, Appl. Phys. Lett. 63. 949 (1993).

C. R. Bolognesi, I. Sela, J. Ibbetson, B. Brar, H. Kroemer, and J. H English. J. Vac. Sci. Technol. B 11. 868 (1993)

${ }^{6} \mathrm{~K}$. Brennan and K. Hess, Solid-State Electron 27. 347 (1984): H. J. Lee and J. C. Woolley, Can. J. Phys. 59. 1844 (1981); G. A. Sai-Halasz. L. L. Chang, J.-M. Welter, C.-A. Chang. and L. Esaki, Solid State Commun. 27, 935 (1978)

${ }^{7}$ We use lattice constants of $6.095 \AA$ for GaSb and $6.058 \AA$ for $\ln A$ s. from A. G. Milnes and A. Y. Polyakov, Solid-State Electron. 36, 803 (1993). For the present case of coherently strained InAs layers on GaSb, the InA lattice constant in the growth direction is $6.058^{3} / 6.095^{2}=5.985 \AA$. assum ing conservation of unit cell volume. Averaging over a (14.8) bilayer (InAs. GaSb) superlattice then yields an average lattice constant of 6.025 $\AA$, and bilayer spacing of $3.012 \AA$

${ }^{8}$ P. Mårtensson and R. M. Feenstra. Phys. Rev. B 39, 7744 (1988)

J. A. Stroscio and R. M. Feenstra, in Methods of Experimental Physics. Scanning Tunneling Microscopy, Vol. 27, edited by J. A. Stroscio and W. J. Kaiser (Academic. Boston, 1993), Chap. 4

${ }^{10}$ R. M. Feenstra and J. A. Stroscio, J. Vac. Sci. Technol. B 5, 923 (1987). "M. McEllistrem, G. Haase, D. Chen, and R. J. Hamers, Phys. Rev. Lett. 70, 2471 (1993)

${ }^{12}$ S. M. Goodnick, R. G. Gann, J. R. Sites, D. K. Ferry, C. W. Wilmsen, D. Fathy, and O. L. Krivanek. J. Vac. Sci. Technol. B 1, 803 (1983).

${ }^{13}$ T. Ando, A. B. Fowler, and F. Stern. Rev. Mod. Phys. 54, 437, 505 (1982).

${ }^{14}$ J. R. Meyer, D. J. Arnold, C. A. Hoffman, and F. J. Bartoli, Appl. Phys Letc. 58, 2523 (1991).

${ }^{15}$ H. Cartensen, R. Manzke, 1. Schäfer, and M. Skibowski, in Proceeding of the 18th International Conference on the Physics of Semiconductors, edited by O. Engström (World Scientific, Singapore. 1987), p. 125

${ }^{16}$ D. Z.-Y. Ting, E. T. Yu, and T. C. McGill. Phys. Rev. B 45, 3583 (1992) Theoretical estimates in the present work were made using InSb-like in terfaces (electron subband energies are $0.02-0.03 \mathrm{eV}$ smaller if GaAs-like interfaces are used).

${ }^{17}$ A. Ourmazd, D. W. Taylow, J. Cunningham, and C. W. Tu, Phys. Rev. Lett. 62, 933 (1989).

${ }^{18}$ C. A. Warwick and R. F. Kopf, Appl. Phys. Lett. 60, 386 (1992).

${ }^{19}$ D. S. Katzer, D. Gammon, and B. V. Shanabrook. J. Vac. Sci. Technol. B 10, $800(1992)$ 